\title{
Coupling Single NV Centers in Diamond to Optical Microcavities
}

\author{
Kai-Mei Fu, ${ }^{1, \dagger}$ Charles Santori, ${ }^{1}$ Sean M. Spillane, ${ }^{1}$ David Fattal, ${ }^{1}$ \\ Qianfan Xu, ${ }^{1}$ Marco Fiorentino, ${ }^{1}$ Raymond G. Beausoleil, ${ }^{1}$ Paul E. \\ Barclay, ${ }^{2}$ Oskar Painter ${ }^{2}$ \\ ${ }^{1}$ Hewlett-Packard Laboratories, 1501 Page Mill Rd., Palo Alto, CA 94304, USA \\ ${ }^{2}$ California Institute of Technology, Pasadena, CA 91125, USA \\ ${ }^{\dagger}$ kai-mei.fu@hp.com
}

\begin{abstract}
Coupling of the $\mathrm{NV}^{-} \mathrm{ZPL}$ to a silica microcavity and tapered fiber is demonstrated at cryogenic temperatures. Coupling to a high-Q cavity should enhance the usefulness of the $\mathrm{NV}^{-}$for quantum information applications.

(c) 2008 Optical Society of America

OCIS codes: (270.1670) Coherent optical effects; (270.5585) Quantum information and processing
\end{abstract}

The negatively charged nitrogen-vacancy $\left(\mathrm{NV}^{-}\right)$center in diamond is a promising candidate for several quantum information (QI) applications including the quantum repeater [1,2]. The strengths of the $\mathrm{NV}^{-}$system include an optically accessible single electron spin and the ability to transfer the electron state to a long-lived nuclear state [3]. However, the utility of the $\mathrm{NV}^{-}$center for most QI applications is limited unless the center can be coupled to an optical microcavity. Many QI applications explicitly require cavity coupling [4, 5]. Additionally, cavity coupling will increase the zero-phonon line (ZPL) photon collection efficiency which according to optical transition selection rules [6] may enable single-shot electron-spin readout. An increase in ZPL collection will also increase the probability of success in entanglement schemes based on single-photon detection [1]. In this work we demonstrate the coupling of the ZPL from a single $\mathrm{NV}^{-}$to a silica microdisk into a tapered fiber at liquid helium temperatures. This work differs from previous work [7] in two important ways. First we are able to positively identify the emitter as a single $\mathrm{NV}^{-}$center through its photoluminescence (PL) signature and an auto-correlation $\mathrm{g}^{(2)}$ measurement. Second we are able to couple the PL out of the cavity into a single-mode waveguide. Efficient particle-cavity and cavity-waveguide coupling should lead to the realization of a "one-dimensional atom" as needed for CQED applications [8].

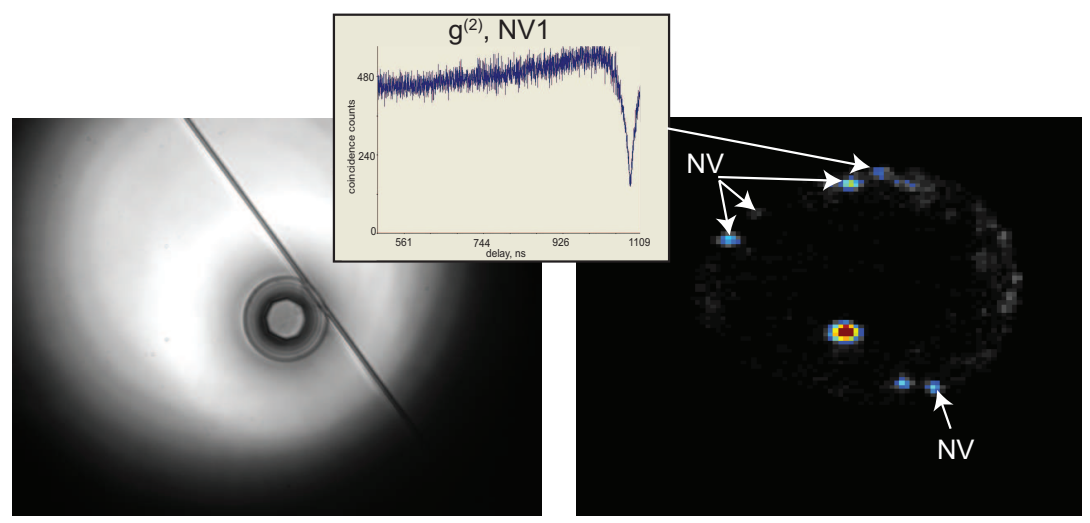

a)

b)

Fig. 1. a) 20 micron silica microdisk with tapered fiber. b) PL scan of silica microdisk with deposited diamond nanoparticles. Several $\mathrm{NV}^{-}$centers are identified. Inset: Autocorrelation measurement demonstrates that $\mathrm{NV} 1$ is a single NV center.

The disks used in this experiment were 20 micron thermal $\mathrm{SiO}_{2}$ microdisks fabricated using standard optical lithography, wet etching, and dry etching. An image of the fiber-disk set-up is shown in Fig. 1a and is similar in geometry to Ref. [9]. The initial quality factor (Q) of these disks was 40000 before the particle deposition. Commercial natural diamond nanoparticles were sonicated in IPA and centrifuged to obtain particles of diameter $<100 \mathrm{~nm}$. The microdisk sample was then dipped into the diamond suspension and dried on a hot plate. During the drying process, the diamond nanoparticles moved to the edge of the disks. Approximately 5-10 particles were deposited per disk. 


\section{JMB3.pdf}

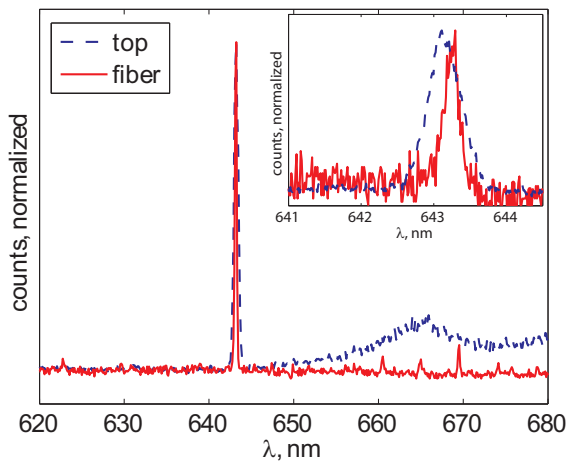

a)

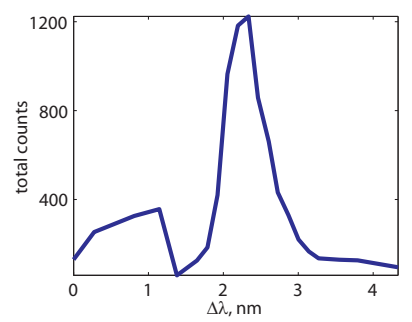

b)

Fig. 2. a) Dashed line: PL from NV1 collected from the top of the microdisk. Solid line: PL from NV1 collected through the fiber. Inset: High resolution spectra. b) Intensity of the $\mathrm{NV}^{-}$emission collected through the fiber as a function of cavity tuning.

In order to identify particles with $\mathrm{NV}^{-}$centers, spatially resolved PL scans of the microdisk were performed. A $100 \mu \mathrm{W}, 532 \mathrm{~nm}, 1 \mu \mathrm{m}$ laser spot was scanned across the surface of the disk as PL in the phonon sideband region (650$800 \mathrm{~nm}$ ) was collected from the top of the disk and detected with a single photon counting module. A scan showing several diamond nano-particles is shown in Fig. 1b. Once identified, PL spectra were obtained of the $\mathrm{NV}^{-}$centers. ZPL linewidths ranged from 0.5 to several THz. The dashed curve in Fig. 2a shows the PL spectrum of NV1 taken from the top of the disk. A $\mathrm{g}^{(2)}$ measurement (Fig.1b inset) demonstrates that this particle contained a single $\mathrm{NV}^{-}$.

In order to demonstrate $\mathrm{NV}^{-}$coupling to the cavity, a tapered fiber was brought into contact with the edge of the disk. After particle deposition and fiber contact, the Q of the cavity degraded significantly to 2700 . The particle was excited from the top of the disk with the $532 \mathrm{~nm}$ laser while PL was collected through the tapered fiber. The cavity was then tuned onto the $\mathrm{NV}^{-}$resonance by allowing a small amount of $\mathrm{N}_{2}$ gas into the cryostat. The gas freezes onto the surface of the cavity, shifting its resonance to longer wavelength. A spectrum of the NV1 PL collected through the fiber with the cavity on resonance is given by the solid line in Fig. 2a. A high resolution spectrum (Fig. 2a inset) shows that the cavity linewidth is narrower than the $\mathrm{NV}^{-}$linewidth for this particular cavity- $\mathrm{NV}^{-}$pair. In Fig. $2 \mathrm{~b}$ the intensity of the NV line as a function cavity tuning is plotted.

In summary we have demonstrated coupling of a single $\mathrm{NV}^{-}$center to an optical microcavity coupled to a tapered fiber at cryogenic temperatures. Due to the low $\mathrm{Q}$ and broad $\mathrm{NV}^{-}$linewidths of the current system, efficient coupling (Purcell factor approaching unity) is still not achieved. However, higher Q cavities with the current geometry should be attainable is we use fewer particles and fiber couple to the disk without actual contact. Additionally, higher quality $\mathrm{NV}$ centers [10] can be used. These improvements will bring us closer to efficient collection of the $\mathrm{NV}^{-} \mathrm{ZPL}$ emission as needed for quantum information processing schemes.

This work was supported by DARPA and the Air Force Office of Scientific Research through AFOSR contract no. FA9550-07-C-0030.

\section{References}

1. L. Childress, J. M. Taylor, A. S. Sorensen and M. D. Lukin, "Fault-tolerant quantum repeaters with minimal physical resources and implementations based on single-photon emitters," Phys. Rev. A 72, 052330-1-16 (2005).

2. S. C. Benjamin, D. E. Browne, J. Fitzsimons, and J. J. L. Morton, "Brokered graph-state quantum computation," New J. of Phys. 8141 (2006).

3. F. Jelezko, T. Gaebel, I. Popa, M. Dormhan, A. Grueger and J. Wrachtrup, "Observation of coherent oscillation of a single nuclear spin and realization of a two-qubit conditional quantum gate, ” Phys. Rev. Lett. 93130501 (2004).

4. T. D. Ladd, P. van Loock, K. Nemoto, W. J. Munro and Y. Yamamoto, "Hybrid quantum repeater based on dispersive CQED interactions between matter qubits and bright coherent light," New J. of Phys. 8184 (2006).

5. W. Yaho, R.-B. Liu and L. J. Sham, “Theory of control of the spin-photon interface for quantum networks," Phys. Rev. Lett. 95, 30504 (2005).

6. Ph. Tamarat, N. B. Manson, R. L. McMurtie, A. Nitsovtsev, C. Santori, P. Neumann, T. Gaebel, F. Jelezko, P. Hemmer and J. Wrachtrup, "The excited state structure of the nitrogen-vacancy center in diamond," cond-mat/0610357

7. Y.-S. Park, A. K. Cook and H. Wang, "Cavity QED with diamond nanocrystals and silica microspheres," Nano Lett. 6, 2075 (2006)

8. Q. A. Turchette, R. J. Thompson and H. J. Kimble, “One-dimensional atoms, ” App. Phys. B 60, S1 (1995)

9. K. Srinivasan, O. Painter, A. Stintz, and S. Krishna, "Single quantum dot spectroscopy using a taper waveguide near-field optic, " App. Phys. Lett. 91, 91102 (2007)

10. F. Jelezko, I. Popa, A. Gruber, C. Tietz, J. Wrachtrup, A. Nizovtsev and S. Kilin, "Single spin states in a defect center resolved by optical spectroscopy," Appl. Phys. Lett. 81, 2160 (2002). 